\title{
Deposition and optimization of thin lead layers for
}

\section{superconducting accelerator photocathodes}

Jerzy Lorkiewicz ${ }^{1}$, Robert Nietubyć ${ }^{1}$, Marek Barlak ${ }^{1}$, Robert Mirowski ${ }^{1}$, Andrzej Bartnik ${ }^{2}$, Jerzy Kostecki², Jacek Sekutowicz ${ }^{3}$, Aneta Malinowska ${ }^{1}$, Peter Kneisel $^{4}$ and Jan Witkowski ${ }^{1}$

${ }^{1}$ National Centre for Nuclear Research (NCBJ), 7 A. Soltana, PL 05-400 Otwock, Poland

${ }^{2}$ Institute of Optoelectronics, Military University of Technology (IOE-MUT),

2 S. Kaliskiego, PL 00-908 Warsaw, Poland

${ }^{3}$ Deutsches Elektronen Synchrotron (DESY), 85 Notkestrasse, D 22-607 Hamburg, Germany

${ }^{4}$ Thomas Jefferson National Accelerator Facility (TJNAF), Newport News, VA, USA

E-mail: jerzy.lorkiewicz@,ncbj.gov.pl

\begin{abstract}
Selection of UHV arc deposition system and recrystallization method was done to optimize smoothness and thickness of thin-layer lead cathodes for superconducting niobium electron injectors. Non-filtered arc system was chosen to deposit $\mathrm{Pb}$ films on niobium. Then the films undergo melting and recrystallization by treating them with pulsed argon ion beams in a rod plasma injector.
\end{abstract}

PACS numbers: 81.15.-z, 68.55.-a, 68.35.Np 


\section{Introduction}

A concept of a $\mathrm{Nb}-\mathrm{Pb}$ hybrid superconducting radio frequency (SRF) niobium photo-injector of electrons with a thin-layer photocathode of lead was proposed in the last decade [1-3]. It was destined for linear superconducting accelerators which provide typically up to $1 \mathrm{~mA}$ average current in $10^{5} \mathrm{~Hz}$ repeated, $1 \mathrm{nC}$ bunches to a free electron laser (FEL) undulator. The lead photocathode can be prepared by deposition on the back wall of a RF cavity using ultra high vacuum (UHV) cathodic arc coating. This deposition method is distinguished by absence of working gas impurities, high degree of ionization of metallic plasma and possible control of the energy of lead ions incoming to a target by applying dc bias to it. The usefulness of the photocathodes reached by this method was confirmed by a proof-ofprinciple test in laboratory conditions reported in [4]. Such photocathode, excited with a laser ultraviolet beam of $195 \mathrm{~nm}$ wavelength, emitted photoelectrons with quantum efficiency (QE) pretty close to its theoretical value $\left(5.410^{-3}\right)$. These measurements indicated that assuring a proper film smoothness and homogeneity at sufficiently high thickness is necessary to operate a gun of this type.

The morphology and microstructure of a lead thin layer after cathodic arc deposition is strongly determined by the presence of micro-droplets coming from erosion of the cathode in a coating device. At RF field gradient in accelerating structure up to ca. $40 \mathrm{MV} / \mathrm{m}$ droplets and other protruding surface irregularities can disturb locally the field distribution and initiate field emission or dark current. In addition, they can influence adversely the photoemission process and reduce QE. The reduction of droplets population can be performed by lead plasma flux filtering during the deposition [5] or by the film annealing and recrystallization after coating. The paper presents the studies dedicated to optimization of post-deposition 
treatment including pulsed EUV, and plasma irradiation It is expected that the photocathodes prepared in a such optimized way will be further examined in terms of resonant quality and quantum efficiency in a TESLA-like electron injector.

\section{2. $\mathrm{Pb}$ film deposition}

Vacuum arc devices used in the early stage of NCBJ photocathode group activity included the following filters of micro-droplets: diaphragms in a "compact" arc deposition system introduced directly into a 1.6-cell niobium cavity (Fig. 1) and a 60o knee-type magnetic filter (Fig. 2). The former was used to coat a $3.5 \mathrm{~mm}$ in diameter, thin-film lead photocathode directly on the rear wall of a radio frequency (RF) cavity. The deposition device was contained in a grounded stainless steel capsule mounted inside the cavity. The top part of the capsule was terminated with a niobium mask placed at a short distance $(<1 \mathrm{~mm})$ from the back wall. The position and size of a circular opening in the mask determined the position and size of the photocathode to be deposited. To intercept lead droplets a system of additional niobium diaphragms was installed between the arc cathode and the mask. Using of the diaphragms resulted in reduction of droplets density in a film (to less than 100 droplets $/ \mathrm{mm} 2$ ) at a cost of presence of peeling products in the layer, detached from lead deposits on diaphragms. They could readily initiate field emission or dark current. In addition arc current and plasma flow were attenuated by the diaphragms which reduced lead deposition rate to merely $20-30 \mathrm{~nm} / \mathrm{min}$. It resulted in low final film thickness (ca. $100 \mathrm{~nm}$ ) as the deposition time is limited by a relatively short life-time of the arc cathode. Because of a small distance between the mask and the wall no dc bias could be applied between them. It precluded the possibility of rising the film density and adhesion by energetic deposition. In spite of promising results of preliminary tests not all photo-cathodes obtained with this system 
survived the standard preparatory procedures applied to TESLA-type cavities including the buffered chemical polishing (BCP) [6] and high pressure rinsing (HPR).

Those disadvantages and other logistic problems led to the cavity reconstruction in the area of the photocathode. $\mathrm{Pb}$ deposition was then performed on a separate, niobium plug (mounted inside the target shown in Fig. 2). The coated plug was next installed in the centre of the cavity back wall. The arc system used for lead deposition was equipped with a kneetype magnetic filter. In this filter the plasma stream emitted from an arc cathode is bent by 60o before reaching the target. It is accomplished by guiding the plasma in a magnetic field with curved lines generated by a coil wrapped around an arc-shaped plasma duct (Fig. 2). Most of the droplets hit the duct wall because of their large mass-to-charge ratio compared with plasma ions. A small fraction of them, however, used to scatter from the walls and reach the coated surface. With this system it was possible to bias the target housing the plug at a negative dc voltage of $70 \mathrm{~V}$ to increase ions energy. Deposition rate of up to $200 \mathrm{~nm} / \mathrm{min}$ resulting in thickness up to ca. $1 \mu \mathrm{m}$ were obtained. Droplets density in a layer was substantially lower than $100 \mathrm{~mm}-2$. Satisfactorily low value of dark current equal to $150 \mathrm{nA}$ was measured in a test injector. On the other hand the corresponding QE values were not reproducible. Scattered values have been measured due to excessive carbon content.

We decided to increase substantially deposition rate and the film thickness on the plug at a cost of filtering removal during deposition. It was accomplished using a short, straight UHV arc system with the arc cathode and the negatively biased target placed at a distance of $10 \mathrm{~cm}$ (Fig.3). The resulting lead deposition rate was $3 \mu \mathrm{m} / \mathrm{min}$. at an arc current $25 \mathrm{~A}$. Nonfiltered lead layer contains typically some tenths of thousands of droplets per $\mathrm{mm} 2$, congealed in forms of hemi-spheres or craters sized from $1 \mu \mathrm{m}$ to $50 \mu \mathrm{m}$ (see Fig. 4). The height of some of them over the film surface exceeds $10 \mu \mathrm{m}$. Therefore, the required film 
flatness has to be obtained by additional heat treatment leading to layer melting and recrystallization.

\section{3. $\mathrm{Pb}$ films heat treatment}

To reach a sufficient quantum efficiency and low dark current from a lead photocathode its surface roughness should not exceed several hundred $\mathrm{nm}$. In order to find an effective way of removing micro-droplets and achieving appropriate flatness a series of tests were performed with melting lead layers deposited in the short arc system on polycrystalline planar niobium samples sized $10 \times 10 \times 2.8 \mathrm{~mm} 3$. These samples were of high-purity niobium routinely used for manufacturing of superconducting RF cavities, characterized with residual resistivity ratio (RRR) higher than 300. Prior to $\mathrm{Pb}$ coating they were polished with diamond paste, rinsed in ultrasonic bath in dry alcohol and next - in de-ionized water, dried in pure argon blow. Two of these samples were lead-coated up to $1 \mu \mathrm{m}$ and two other samples - to $12 \mu \mathrm{m}$, (see the microscope images in Fig. 4) by applying short (7 s) UHV arc pulses separated by time intervals of $5 \mathrm{~min}$. This coating regime was chosen to avoid overheating and excessive erosion of the arc cathode. After deposition the films were melted with beams of extreme ultraviolet radiation (EUV) in vacuum or with argon plasma ion beams. The resulting changes in surface morphology and composition were observed using SEM.

Focused EUV radiation was generated by a Xe puff irradiated by the Nd YAG pump laser at IOE-MUT [7]. The wavelength ranged from 8 to $70 \mathrm{~nm}$ (with intensity maximum at $\lambda=11 \mathrm{~nm}$ and with ca. $75 \%$ of the integrated spectral intensity corresponding to $8 \mathrm{~nm}<\lambda<17$ $\mathrm{nm}$ ) at a standard for this device pulse duration of $5 \mathrm{~ns}$ and repetition rate $10 \mathrm{~Hz}$. The typical absorption length of EUV radiation of this source in lead was ca. $20 \mathrm{~nm}$. The available fluence on the beam axis was equal $30 \mathrm{~mJ} / \mathrm{cm} 2$. The fluence drops by a factor larger than 2 at 
$1 \mathrm{~mm}$ from the axis [7]. Two of the niobium samples with $1 \mu \mathrm{m}$ and $12 \mu \mathrm{m}$ lead films of morphology shown in Fig. 4 were irradiated with EUV pulsed beam. SEM images of the film surface after coating and after applying 1, 2, 5, 10, 20 and 40 EUV pulses were recorded. The SEM image in Fig. 5a which corresponds to 40 pulses applied to the $1 \mu \mathrm{m}$ thick layer reveals a process of lead coagulation on a non-coated niobium surface (visible as a dark background). It is associated with formation of ca. $0.5 \mu \mathrm{m}$ in diam. lead spheres which were gradually removed from the substrate with the growing number of EUV pulses. The tests indicated lack of lead layer adhesion to the niobium surface which can be attributed to insufficient wettability. There is no direct experimental data in the literature concerning niobium wetting by liquid lead. As a rule, low melting-point metals (e.g Pb) show poor adhesion to many metallic surfaces and high contact angles at temperatures close to their melting points [8]. 12 $\mu \mathrm{m}$ thick lead layer surface was modified after applying 40 EUV pulses in a way shown in Fig. 5b. As expected, it was observed on SEM images that thick layer remained continuous. Selective melting of its surface did not improve its flatness.

A second approach to melting and recrystallization of a lead film on niobium was performed using IBIS rod plasma injector at NCBJ [9-10]. The injector is capable of generating argon plasma ion pulses with fluence from 1 to $7.8 \mathrm{~J} / \mathrm{cm} 2$ at a typical pulse duration time of $1 \mu \mathrm{s}$. The consecutive pulses are separated in time by $3 \mathrm{~min}$. Thus, the effects of successive pulses do not accumulate. As it was estimated using Energy Transport in Laser Irradiated Targets (ETLIT) code (see [10]), the fluence of a single $1 \mu$ s long ion pulse should be lower than $4 \mathrm{~J} / \mathrm{cm} 2$ to avoid melting of bare, bulk niobium. Calculations confirmed that pure, bulk lead is melted down to ca. $5 \mu \mathrm{m}$ from its surface as a result of applying a single $3 \mathrm{~J} / \mathrm{cm} 2$ ion pulse of this duration. It can be deduced that a $1 \mu \mathrm{m}$ thick $\mathrm{Pb}$ layer on niobium is completely melted (without melting the underlying niobium), whereas a $12 \mu \mathrm{m}$ 
thick lead film is melted only partially after irradiating with a single ion pulse of this duration and energy density.

Four $1 \mu \mathrm{s}$ long ion pulses of $3 \mathrm{~J} / \mathrm{cm} 2$ fluence were applied in IBIS to $1 \mu \mathrm{m}$ and $12 \mu \mathrm{m}$ thick lead layers on niobium. Irradiation of the thinner one resulted in sufficiently flat but incomplete (perforated) layer (Fig. 5c) due to led melting and poor wettability. In Fig. 5c roughly one third of the surface is deprived of lead coating. Irradiation of $12 \mu \mathrm{m}$ layer resulted in melting and disappearance of all extrusions (droplets and craters) with diameters up to $20 \mu \mathrm{m}$, while the few remnants of larger structures were strongly flattened and had mitigated edges and contours (Fig 5d). Objects with such aspect do not initiate dark current. The film surface remained continuous in this case.

$20 \mathrm{keV}$ electron beam induced X-ray signal was used (within EDS option of EVO 10 MA scanning electron microscope) to reveal the elemental composition of $\mathrm{Pb}$ layer averaged over the surface area shown in Fig. 5d. It comprises: $94 \% \mathrm{~Pb}, 2 \% \mathrm{Nb}, 1.7 \% \mathrm{O}$ and $2 \% \mathrm{C}$ by weight. Such a purity, including substantially lower oxygen and carbon contents, is significantly better than that of $100 \mathrm{~nm}$ thick photocathode reported in [1] $(90.4 \% \mathrm{~Pb}, 6.2 \% \mathrm{C}$ and $3.3 \% \mathrm{O}$ ). The latter was deposited using a 30o knee-type filter. Further reduction of oxides and carbon contents is routinely obtained by using laser cleaning inside the superconducting e- injector before testing QE.

\section{Simulation of heating up lead films}

Experience gained from the performed plasma irradiation led us to the conclusion that it is more adequate than the EUV one. In order to examine possibility of lead surface melting and droplets removal or flattening, rough heat transfer calculation was performed using FEMM code which applies finite element method [11] We considered typical surface morphology 
resulted from arc deposition objects occurring on the surface: droplets of various sizes and shapes, craters, edges as well as flat and smooth area, see Fig 6. Energy dose delivered with fluence of $3 \mathrm{~J} / \mathrm{cm} 2$ in $1 \mu \mathrm{s}$ has been assumed. During irradiation only absorption was taken into consideration. Neither radiative nor convective heat losses were taken into account. Only radiation cooling with emissivity equal to 0.5 was assumed afterwards. In order to approximate ions penetration depth of several nanometers we calculated the provided energy as a heat flux through upper surface of the film, accounting for diversified exposition angle.

Evaluation showed that droplets due to hindered heat transfer towards bulk material accumulate significantly more energy than flat regions. The width of droplet junction to the bulk film is critical for energy transfer. Narrow junction leads to vaporization of droplet material. Our calculations showed that all droplets and a layer down to the depth of about 10 $\mu \mathrm{m}$ is melted. According to our evaluation this condition lasts several microseconds, however including the convection results in reduction of melted layer and time needed for solidification. Results of performed evaluation are consistent with the observed changes in surface morphology and confirmed that IBIS plasma irradiation preferably ablates the droplets and allows surface recrystallization. Therefore it is a propitious treatment for surface flattening.

\section{Conclusions and future plans}

Studies on optimizing smoothness and thickness of lead films on niobium substrate are just the first step towards elaborating effective procedure of preparing superconducting einjectors with reproducible working parameters. Fast lead deposition in a short, straight, planar vacuum arc system followed by melting and recrystallization of the surface layer in the IBIS rod plasma injector was selected as the most promising way to reach this goal. The 
ultimate check of the usefulness of this approach, however, must include direct determination of QE and dark current at typical RF field gradient. To this end a suitable experimental teststand is currently being completed at NCBJ to conduct these measurements, which include the possibility of preliminary cleaning of lead films with pulsed UV laser beam.

Acknowledgments

Investigations were supported with European Coordination in Accelerator Research and Development, a part of FP7

References

[1] Rao T, Smedley J, Warren J, Kneisel P, Nietubyc R and Sekutowicz J 2010 Proc. 1st International Particle Conference IPAC10 (Kyoto, Japan), THPECO 204086

[2] Smedley J, Rao T and Sekutowicz J 2008 Phys. Rev.i Spec. Topics - Accelerators and Beams 11013502

[3] Sekutowicz J et al. 2007 Proc. 22nd Particle Accelerator Conf. PAC07, (Albuquerque, New Mexico, USA) TUPMN0 21962

[4] Langner J et al. 2007 IEEE Trans.Plasma Sci. 35100

[5] Anders A 1999 Surface and Coat.Technol. 120-121 319

[6] Antoine C 2012 Materials and Surface Aspects in the Development of SRF Niobium Cavities, Editorial Series on Accelerator Science XII (Warsaw: Institute of Electronic Systems, Warsaw University of Technology)

[7] Bartnik A, Fiedorowicz H, Jarocki R, Kostecki J, Szczurek M and Wachulak P W 2011 Nucl. Instr. Meth. Phys. Res. A 647125 
[8] Eustathopoulos N, Sobczak N, Passerone A and Nogi K 2005 J. Mater. Sci. 402271

[9] Piekoszewski J, Langner J, Walis L, Werner Z, Pochrybniak C, Czaus K, Ciurapinski A and Białoskórski J 1994 Nukleonika 393

[10] Barlak M 2010 High Intensity Plasma Pulses in Ceramic Wettability Improvement (Otwock-Swierk, Poland: The Andrzej Soltan Institute for Nuclear Studies) in English.

[11] Meeker D www.femm.info 


\section{Figure captions}

Figure 1. "Compact" arc deposition system inside a 1.6-cell RF superconducting electron injector.

Figure 2. Deposition system with a $60^{\circ}$ knee-type filter.

Figure 3. Straight UHV arc deposition system.

Figure 4. Optical microscope images of $\mathrm{Pb}$ layers deposited on $\mathrm{Nb}$ samples in the straight arc system up to $1 \mu \mathrm{m}$ (a) and to $12 \mu \mathrm{m}(\mathrm{b})$.

Figure 5. SEM images of $1 \mu \mathrm{m}(\mathrm{a}, \mathrm{c})$ and $12 \mu \mathrm{m}$ thick (b,d) lead layers after treating with forty pulses of EUV radiation $(a, b)$ and with four $3 \mathrm{~J} / \mathrm{cm} 2$ argon plasma ion pulses $(\mathrm{c}, \mathrm{d})$. Note the scale difference.

Figure 6. Vaporised regions and temperature distribution calculated for single plasma pulse irradiation. Regions; 1 - ablated, 2-6 liquid warmer than $1850 \mathrm{~K}, 1640 \mathrm{~K}, 1225 \mathrm{~K}, 820 \mathrm{~K}$ and $605 \mathrm{~K}$, respectively, 7 -solid 


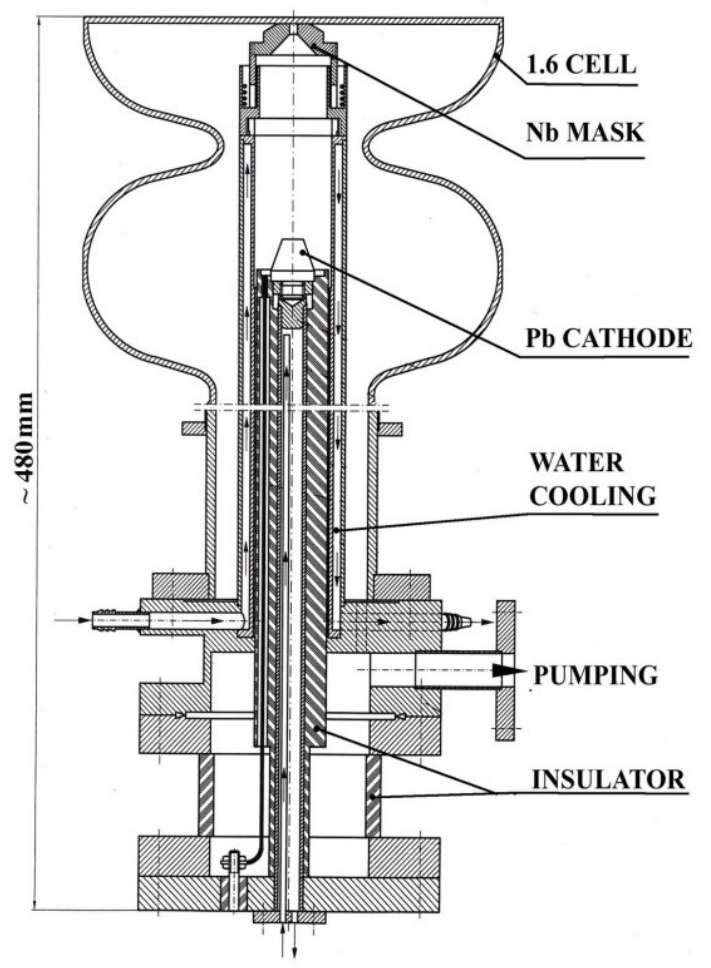

J. Lorkiewicz et al

Figure 1. 


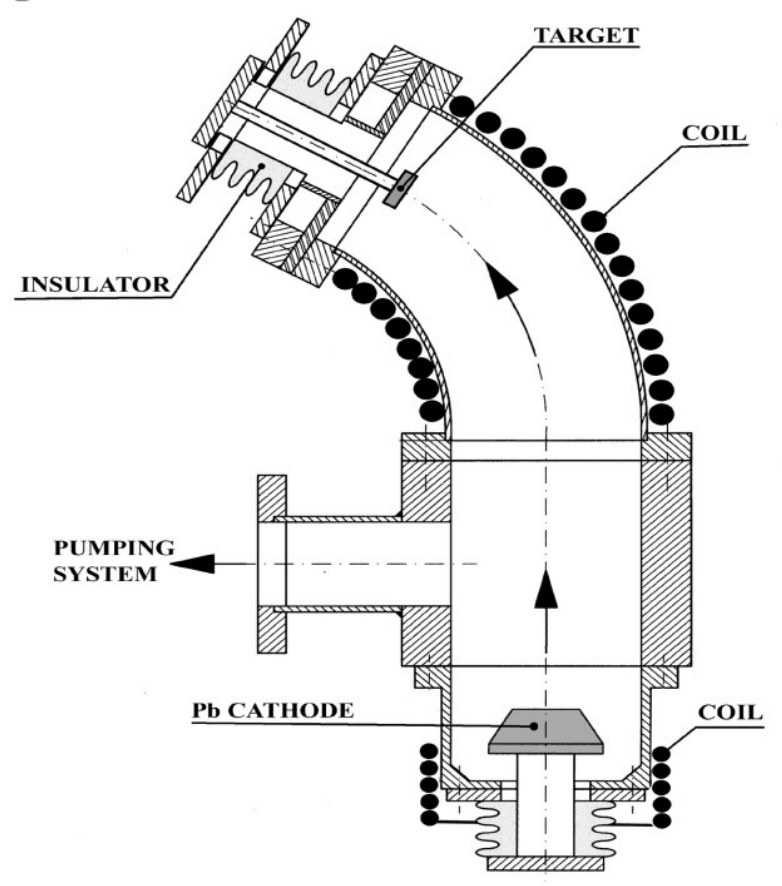

J. Lorkiewicz et al

Figure 2. 


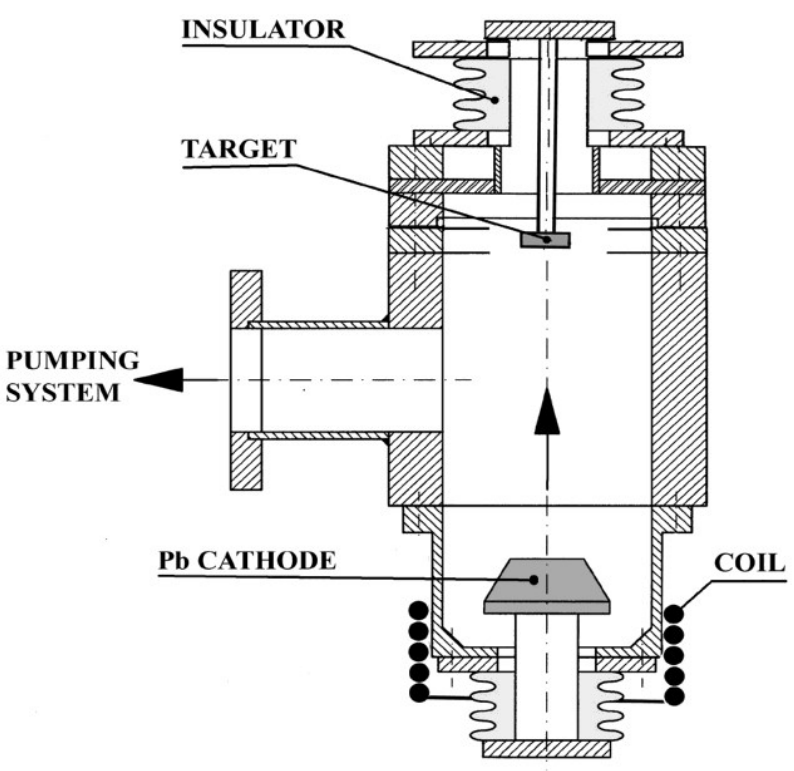

J. Lorkiewicz et al

Figure 3. 

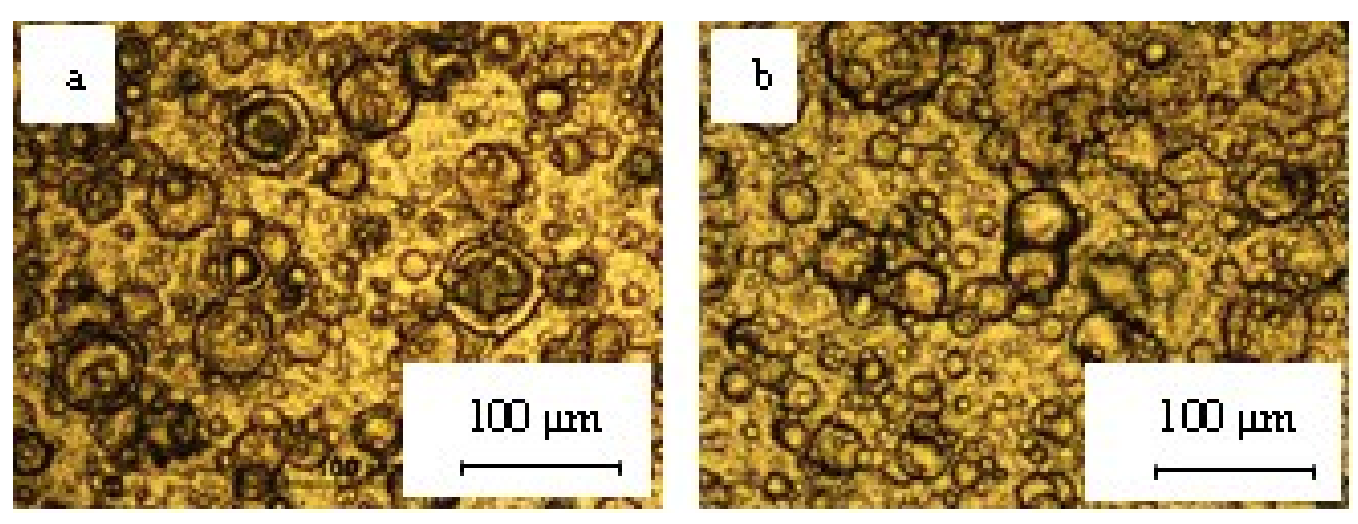

J. Lorkiewicz et al

Figure 4. 


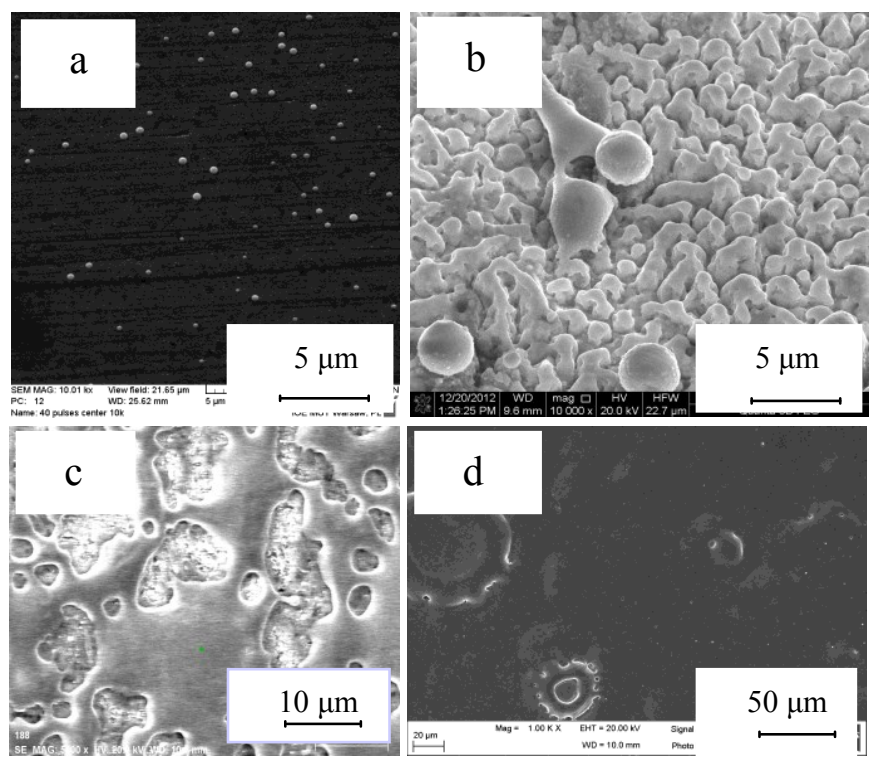

J. Lorkiewicz et al

Figure 5. 


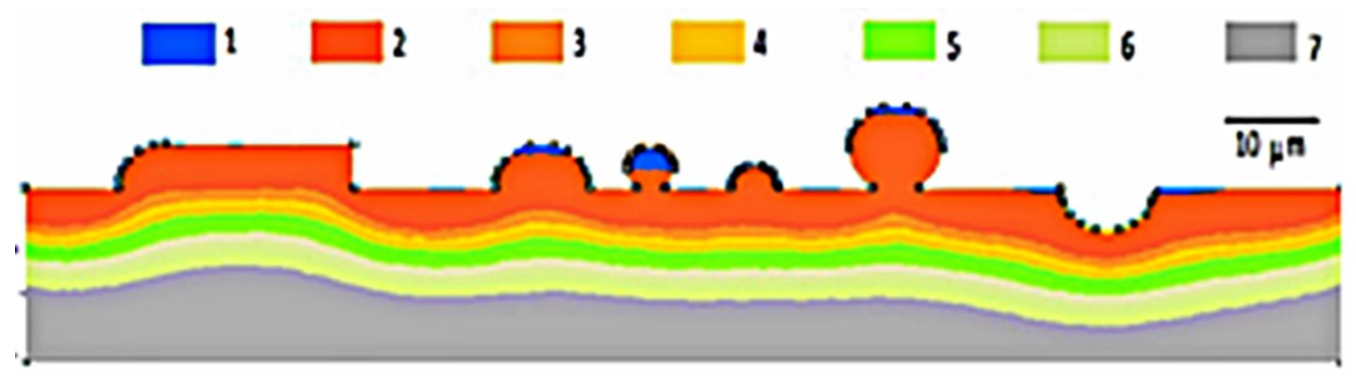

J. Lorkiewicz et al.

Figure 6. 